\title{
Recovery process of a transformer type superconducting fault current limiter
}

\section{AUTHOR(S):}

Shirai, Y; Fujikawa, K; Shiotsu, M; Hatta, H; Muroya, S; Nitta, T

\section{CITATION:}

Shirai, Y ... [et al]. Recovery process of a transformer type superconducting fault current limiter. IEEE TRANSACTIONS ON APPLIED SUPERCONDUCTIVITY 2002, 12(1): 880-884

\section{ISSUE DATE:}

2002-03

URL:

http://hdl.handle.net/2433/50256

\section{RIGHT:}

(c)2002 IEEE. Personal use of this material is permitted. However, permission to reprint/republish this material for advertising or promotional purposes or for creating new collective works for resale or redistribution to servers or lists, or to reuse any copyrighted component of this work in other works must be obtained from the IEEE. 


\title{
Recovery Process of a Transformer Type Superconducting Fault Current Limiter
}

\author{
Y. Shirai, K. Fujikawa, M. Shiotsu, H. Hatta, S. Muroya, and T. Nitta
}

\begin{abstract}
Recovery process of a transformer type superconducting fault current limiter (SCFCL) was investigated by experiments using three-phase test SCFCL designed and made. "Recovery Time" decreases, as "Fault Time" is longer. It is pointed out that the FCL current at the limiting mode affects the cooling process of the secondary wire during the current limiting operation. A simple simulation of the SCFCL based on the heat equation was performed. The simulation results of the "Recovery Time" agree well with the experimental ones. The temperature of the normal zone was estimated by use of the simulation. It decreases even in the current limiting mode and its decreasing rate depends on the limiting current. After a long limiting operation (over $5 \mathrm{~s}$ ), the temperature saturated about $22 \mathrm{~K}$, the secondary coil current is the minimum propagation current of the wire and the "Recovery Time" is quite short and independent of the limiting current.
\end{abstract}

Index Terms-Heat equation, recovery process, recovery time, superconducting fault current limiter.

\section{INTRODUCTION}

$\mathbf{S}$ UPERCONDUCTING fault current limiters (SCFCLs) are expected to make a power system flexible and reliable. Various types of SCFCL have been proposed and studied [1]-[3]. The important features of SCFCL in a power system are the trigger current level, the current limiting impedance and the recovery time. We have proposed an SCFCL with adjustable trigger current level and a trial one (single phase), which is a kind of transformer type, was designed and made [4]. Basic tests on the SCFCL were carried out. The basic test results showed that the recovery time, that is, the required zero-current period for successful recovery, is quite short and becomes shorter as the current limiting period is longer [5]. Under these studies, a new three-phase SCFCL of the proposed type was designed and made [6].

In this paper, using one of the three-phase SCFCL, the recovery process was investigated by experiments and computer simulation. The recovery time was measured with various current-limiting periods and the limiting currents. A simple simulation of the SCFCL based on the heat equation was performed to confirm these consideration and to investigate the recovery process of the SCFCL.

\footnotetext{
Manuscript received September 24, 2001. This work was supported in part by the Japan Society for the Promotion of Science under Project JSPS-RFTF97P01004.

Y. Shirai, K. Fujikawa, and M. Shiotsu are with Department of Energy Science and Technology, Kyoto University, Yoshida-Honmachi, Sakyo-ku, Kyoto, 606-8501 Japan (e-mail: \{shirai; fujikawa; shiotsu\} @energy.kyoto-u.ac.jp).

T. Hatta, S. Muroya, and T. Nitta is with Department of Electrical Engineering, the University of Tokyo, 7-3-1, Hongo, Bunkyo-ku, Tokyo, 113-8656 Japan (e-mail: \{hitta; muroya; nitta\} @ asc.t.u-tokyo.ac.jp).

Publisher Item Identifier S 1051-8223(02)03846-0.
}

TABLE I

SPECIFICATION OF TEST SCFCL

\begin{tabular}{llc}
\hline Primary coil & Lenglh & I $80 \mathrm{~mm}$ \\
& Inner diameter & $80 \mathrm{~mm}$ \\
& Number of tums & 360 \\
& Self Indactance & $3.82 \mathrm{mH}$ \\
\hline Secondary coil & Length & $180 \mathrm{~mm}$ \\
& Inner diamcter & $90 \mathrm{~mm}$ \\
& Number of turms & 1.38 \\
& Self Inductanoe & $0.69 \mathrm{mH}$ \\
\hline Mutual inductance & & $1.43 \mathrm{mH}$ \\
\hline
\end{tabular}

TABLE II

SPECIFICATIONS OF SUPERCONDUCTING WIRE FOR SECONDARY WIRE

\begin{tabular}{|c|c|c|}
\hline Fums: & & Seccifcaton \\
\hline \multirow[t]{10}{*}{ Strand } & Strucsure & Mutiflament \\
\hline & Diamcter of strand & $0.126 \mathrm{men}$ \\
\hline & Mactral of matrix & CU-30ANi \\
\hline & & $\mathrm{CuNi} \mathrm{NbT}-4$ 3:1 \\
\hline & Material of filament & $\mathrm{NbT}$ \\
\hline & Diameter of filameat & Q.08 yet \\
\hline & Number of filameat & 441,575 \\
\hline & Twist pitah & $0.86 \mathrm{~mm}$ \\
\hline & Twist drection & Condwise(5) \\
\hline & Invelation & None \\
\hline \multirow[t]{5}{*}{ Twishod wire } & Strucare & 6 strands + Core \\
\hline & Material of eore & Cu:30hani \\
\hline & Diamkter of coec & $0.16 \operatorname{mm}$ \\
\hline & Twist piteh & $3.5 \mathrm{~mm}$ \\
\hline & Twist dinestion & Chorkwise(5) \\
\hline \multirow[t]{2}{*}{ Characketintics } & Crikcal curretir (14) & I80 Aruts (at 4.2K,0T) \\
\hline & Nurmal roistance & $3.18 \mathrm{n} \mathrm{m}$ \\
\hline
\end{tabular}

\section{TEST SCFCL}

The three-phase SCFCL unit contains three SCFCLs of transformer type in one cryostat [6]. The test SCFCL consists of two superconducting coils coupled co-axially. The inner (primary) coil will be connected to a power line. The outer (secondary) coil is short-circuited. The primary coil can be slided with small slide distance in order to calibrate the trigger current level. Both superconducting coils are in the superconducting state in the waiting mode. The reactance of SCFCL is small (leakage reactance). When the fault current reaches the trigger current level, the super-normal transition occurs only in the secondary coil. The reactance of the SCFCL becomes large (almost equal to that of the primary coil) and the fault current is limited. The specifications are listed in Table I. The specifications of the superconducting wire of the secondary coil are shown in Table II. The superconducting wire of the primary coil is made of twisted three $\mathrm{CuNi} / \mathrm{Cu} / \mathrm{NbTi}(3.3: 0.3: 1)$ strands. The designed trigger current level without calibration is 85.7 Arms. 


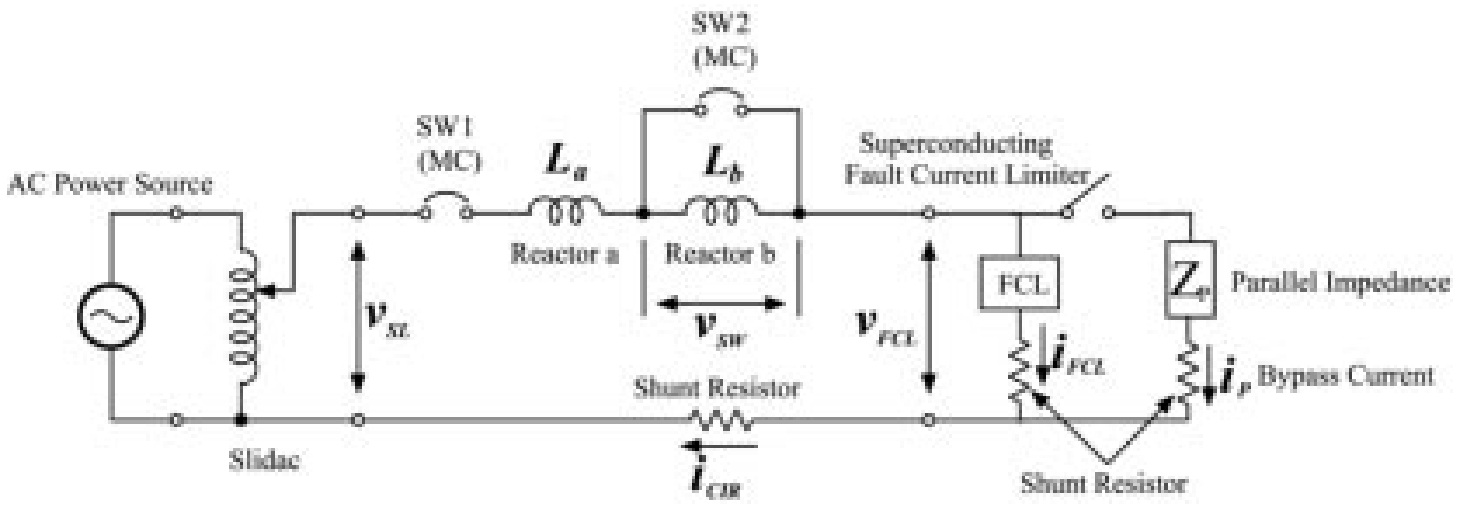

Fig. 1. Experimental circuit for recovery test.

\section{RECOVERY CHARACTERISTICS}

The "Recovery Time" (the required zero current time of the SCFCL for recovery from the current limiting mode to the waiting mode) was measured for various "Fault Time" (the time while the fault current flows through the SCFCL) using the trial single-phase SCFCL [5]. It was confirmed that the "Recovery Time" depends on the "Fault Time" (see Fig. 3: for the old SCFCL). The "Recovery Time" increases with the "Fault Time" of $0 \mathrm{~s}$ to $0.2 \mathrm{~s}$ and has peak value $0.37 \mathrm{~s}$ at around 0.2 s of the "Fault Time." The "Recovery Time" decreases gradually and approaches to a certain value (a few ten ms), as the "Fault Time" is longer.

It is not clear the reason why the "Recovery Time" has the peak value. It was expected that the temperature of the secondary wire at the beginning of recovery process depend on the "Fault Time" and determine "Recovery Time," but it was not clear. The trigger current level of the old SCFCL was degraded from its designed value. It was caused by the current concentration at the contact part between the short circuit copper bar and the superconducting wire at the beginning of the current limitation [7]. The fact may affect the recovery characteristics.

The new 3-phase SCFCL was designed to avoid the current concentration at the contact. The superconducting wire has the same specification of that for the old single-phase SCFCL except the twist pitch of the strand, which is $0.7 \mathrm{~mm}$ for old one, and the twist direction and pitch of the wire, which is counter-clockwise and $2 \mathrm{~mm}$ for old one, respectively. It was confirmed experimentally that the trigger current levels of three SCFCLs are almost agree with the designed value.

Characteristics of "Recovery Time" versus the "Fault Time" are measured using new 3-phase SCFCL. The temperature change of the secondary wire in the recovery process is estimated by the computer simulation.

\section{EXPERIMENT AND DISCUSSION}

\section{A. Experimental Circuit}

An experimental circuit is shown in Fig. 1. A short-circuit switch SW2 (MC: Magnetic Controlled Contact) is connected in parallel to the reactor " $b$ " to simulate a fault. The reactor " $a$ " simulates the transmission line. The inductance $L_{a}$ and $L_{b}$ of the reactors are $2.13 \mathrm{mH}$ and $6.40 \mathrm{mH}$, respectively. The frequency

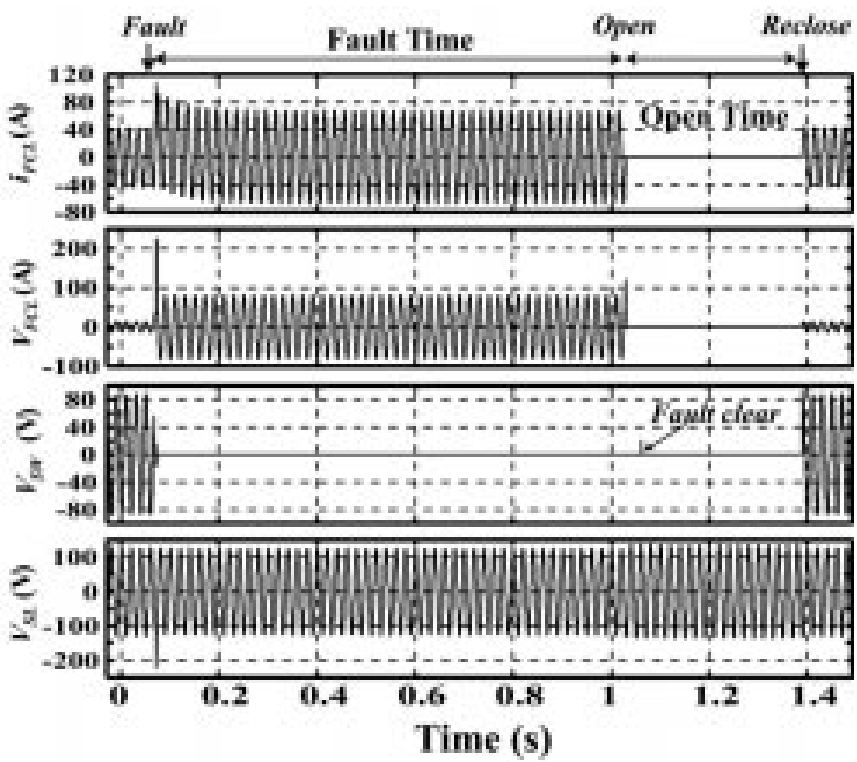

Fig. 2. Wave forms of voltages and currents. (Fault Time $=0.95 \mathrm{~s}$, Open Time $=0.36 \mathrm{~s})$.

of AC power source is $50 \mathrm{~Hz} . Z_{p}$ is a reactor of $2.13 \mathrm{mH}$. The reactance of SCFCL is $0.83 \mathrm{mH}$, which is leakage reactance of the coils), in the waiting mode and is $3.82 \mathrm{mH}$, which is the reactance of the primary coil, in the current limiting mode.

The current $i_{\text {FCL }}$ of the SCFCL, the circuit current $i_{C I R}$, the current $i_{p}$ of the bypass circuit, the voltage $v_{\mathrm{FCL}}$ of the SCFCL, the voltage $v_{\text {SL }}$ of the output voltage of the slidac and the voltage $v_{\text {SW }}$ across the reactor " $b$ " were measured.

\section{B. Experimental Result}

At first, the switch SW1 is closed and the switch SW2 is open. SW2 is closed to simulate a fault. The super-normal transition occurs at only the secondary wire when $i_{\mathrm{FCL}}$ reaches the trigger current level. The SCFCL turns into the current limiting mode. After a moment, SW1 is open to remove the fault circuit and the SCFCL from the power source. "Fault Time" is defined as the time while the fault current flows. Then SW2 is opened to clear the fault. SW1 is re-closed to reconnect the reactors (load) and the SCFCL. "Open Time" is defined as the time while the SCFCL is disconnected from the power source, that is, the zero current period. 


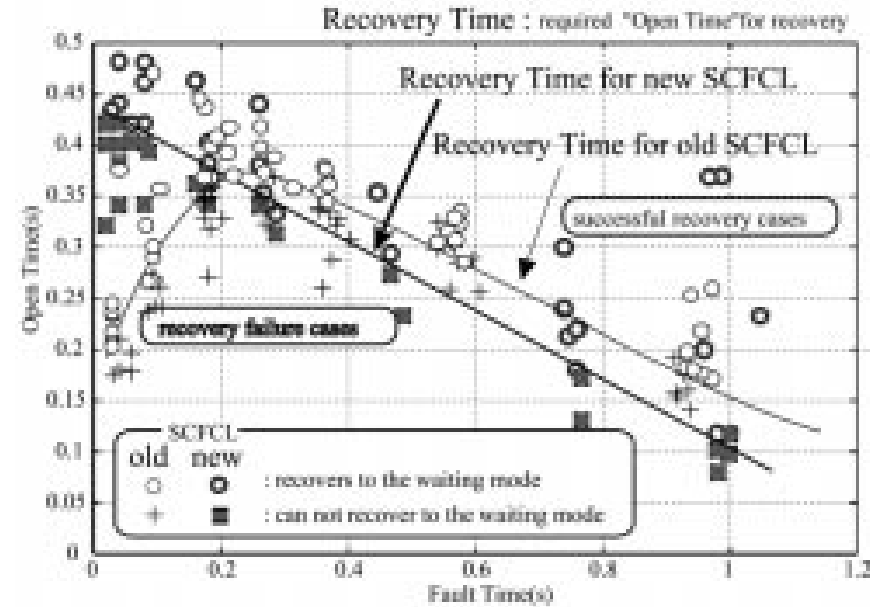

Fig. 3. Recovery Time versus Fault Time for new (3-phase) and old (trial single-phase) SCFCL.

One of the experimental results is shown in Fig. 2. The initial conditions are as follows: $v_{\mathrm{SL}}=91.9 \mathrm{Vrms}, i_{\mathrm{FCL}}=31.1$ Arms. The SCFCL begins to limit the fault current immediately after the fault occurred $(0.09 \mathrm{~s})$. The fault current is limited to be 49.5 Arms. It would be 93.6 Arms without the SCFCL. SW1 is open at $1.04 \mathrm{~s}$ to remove the fault circuit. SW2 is open to clear the fault at $1.07 \mathrm{~s} . \mathrm{SW} 1$ is re-closed at $1.39 \mathrm{~s}$ and the SCFCL recovers to the waiting mode successfully in this case.

\section{Recovery Time}

Experiments with various "Fault Time" and "Open Time" were carried out. Required "Open Time" for successful recovery is measured as a function of "Fault Time." The experimental results are shown in Fig. 3, where the experimental data with old SCFCL are also indicated [5].

Thin and thick solid lines indicate the boundary of two areas of the successful recovery cases and the failure cases for the old SCFCL (single-phase) and the new SCFCL (3-phase), respectively. These lines are regarded as the "Recovery Time." As can be seen from Fig. 3, the "Recovery Time" for new SCFCL has the value of about $440 \mathrm{~ms}$ against the "Fault Time" of about $40 \mathrm{~ms}$, and decreases, as the "Fault Time" is longer. The new SCFCL shows quick recovery characteristics.

Two lines are different while the "Fault Time" is in interval from $0 \mathrm{~s}$ to about $0.2 \mathrm{~s}$. The difference between the old SCFCL and the new SCFCL is a part of the contact between the secondary wire and the short-circuit copper bar [7]. Because the wire of secondary coil of the new SCFCL is the same as that of the old SCFCL, the difference in the "Recovery Time" characteristics in Fig. 3 is caused by the design of the contact. It is expected that heat spot at the contact is generated at the beginning of the current limiting mode in the old SCFCL and the normal zone spreads along the wire. The contact of the new SCFCL was improved to avoid the heat spot. It is considered that the temperature profile along the secondary wire at the beginning of the current limiting is different from that for the old SCFCL.

The "Recovery Time"-characteristics is similar to each other when "Fault Time" is longer than $0.2 \mathrm{~s}$. The influence of the heat spot on the temperature profile diminishes. It can be said

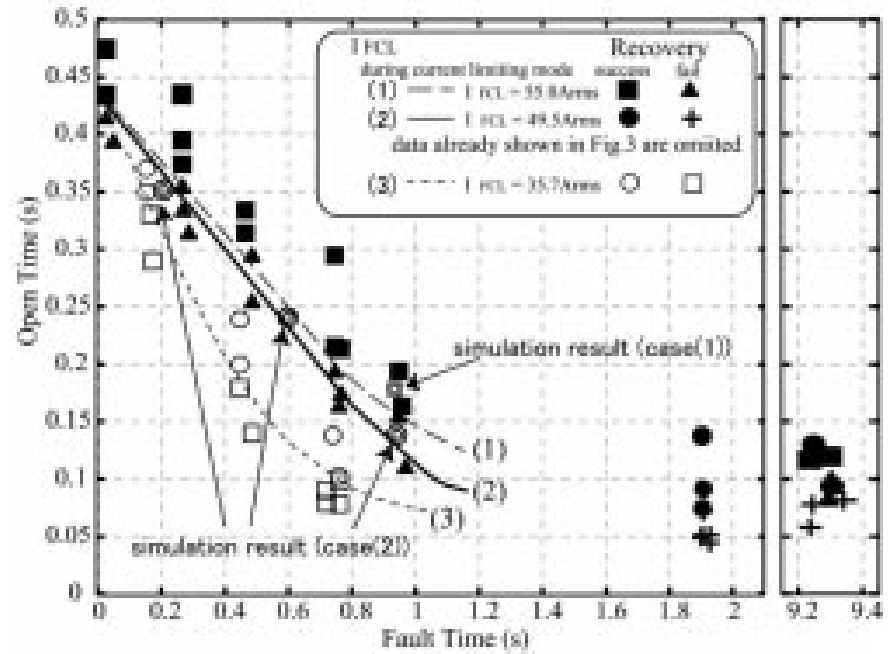

Fig. 4. Required "Open Time" for recovery with FCL current at the limiting mode as a parameter.

that the "Recovery Time" characteristics is mainly determined by the specification of the secondary wire.

\section{Recovery Time and SCFCL Current at the Current Limiting Operation}

The influence of the limiting current on the recovery characteristics is investigated in this section. In the experimental circuit shown in Fig. 1, following cases were set.

1) $L_{a}=3.18 \mathrm{mH}, v_{\mathrm{SL}}=123 \mathrm{Vrms}, Z_{p}$ : open

2) $L_{a}=2.13 \mathrm{mH}, v_{\mathrm{SL}}=91.9 \mathrm{Vrms}, Z_{p}$ : open

3) $L_{a}=2.13 \mathrm{mH}, v_{\mathrm{SL}}=113.8 \mathrm{Vrms}, Z_{p}: 2.13 \mathrm{mH}$.

The FCL current at the fault before the current limiting mode is 95 Arms in every case, and that in the current limiting mode is 1) $55.0,2) 49.5$ and 3) 35.7 Arms, respectively.

Required "Open Time" for successful recovery was obtained as a function of "Fault Time" with FCL current at the limiting operation as a parameter. Fig. 4. shows the experimental result. Experimental data of case 2), which are already shown in Fig. 3, are omitted for convenience. Only the characteristic line is shown. The "Recovery Time" is longer as the FCL current at the limiting mode is higher. This result means that the FCL current at the limiting mode affects the cooling process of the normal zone of the secondary wire during the current limiting operation. However, there is small difference in "Recovery Time" among three cases for shorter than $0.1 \mathrm{~s}$ and around $9.3 \mathrm{~s}$ of "Fault Time." This result is related to the temperature change during the current limiting operation.

\section{E. Recovery Process and Temperature Of Secondary Wire}

In order to estimate the temperature of the normal zone of the wire, computer simulation was carried out for the experimental circuit (Fig. 1). The resistance of the secondary coil is given from an analytical model based on a one-dimensional heat equation of the wire. Temperature dependency of the thermal conductivity, specific heat, resistivity of the materials of the superconducting wire is taken into account [8]-[10].

Two examples of the simulation results for case 2) are shown in Fig. 5. The voltage $v_{\mathrm{FCL}}$ and current $i_{\mathrm{FCL}}$ of the SCFCL, and 

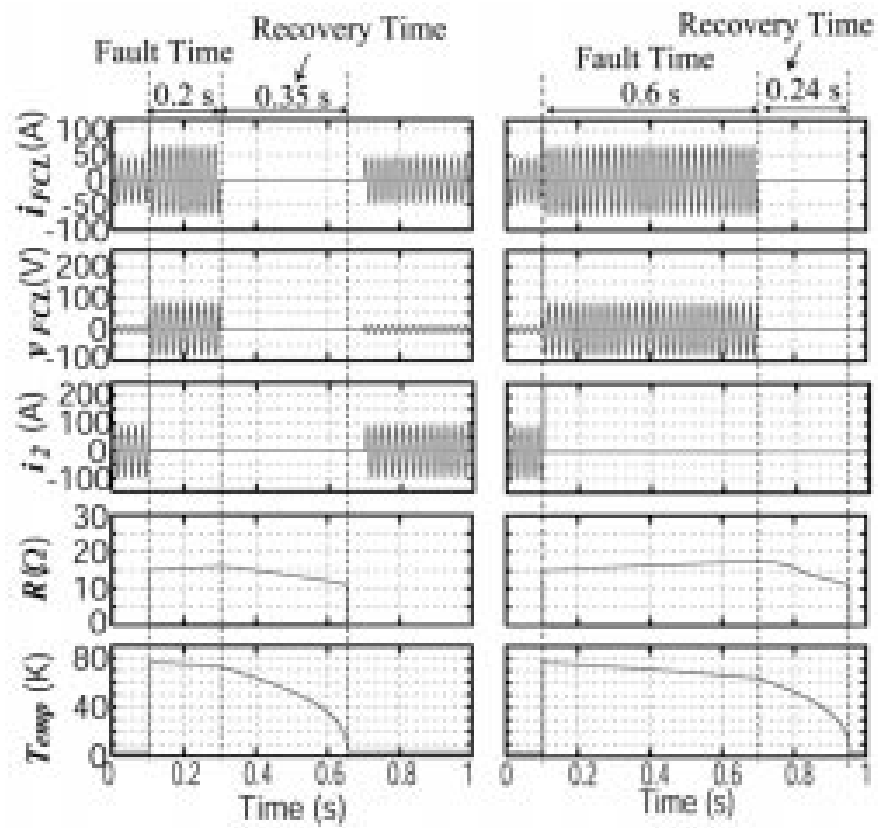

Fig. 5. Simulation result of recovery test [case 2)].

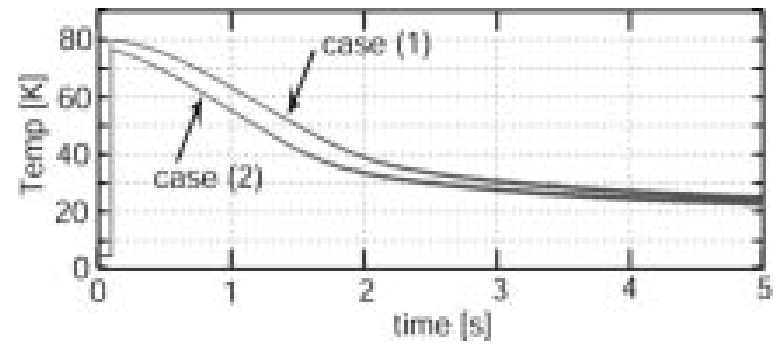

Fig. 6. Temperature change with time of the normal zone of secondary wire in case 1) and in case 2) while the current limiting operation continues.

the current $i_{2}$ of the secondary coil, the resistance $R$ of the secondary coil and the maximum temperature Temp of the wire are shown. The current, temperature and resistance of the secondary coil cannot be measured directly in the experiment. The recovery process is discussed by the experimental and also simulation results. The maximum temperature at the beginning of the current limiting is evaluated to be about $77 \mathrm{~K}$ in case 2 ). The temperature decreases even while the fault current flows through the SCFCL. If all the secondary wire is in the normal state, the resistance $R$ is about $125 \Omega$. Therefore it can be said that only a part of the superconducting wire has resistance (normal zone). Because there is no heat generating power source during the period of "Open Time" in the SCFCL, a normal zone of the superconducting wire is cooled down and disappears at once when Temp reaches the critical temperature, and the SCFCL recovers to its waiting mode after a certain time ("Recovery Time"). The "Recovery Time" depends on the temperature Temp at the beginning of the zero current period, which is a function of FCL current in the current limiting mode. The simulation results of "Recovery Time" for case 1) and 2) are plotted in Fig. 4. They agree well with the experimental results.
Fig. 6 shows the calculated temperature changes in case 1) and 2) when the current limiting operation continues and fault current lasts. Temperature of the normal zone in case 1) is higher than that in case 2), but it becomes close to that in case 2) with time. This result can explain the dependency of the limiting current on the "Recovery Time" shown in Fig. 4.

It was already reported [11] that, after a sufficient time from the fault occurrence, the secondary coil current becomes almost equal to the minimum propagation current of the superconducting wire. The simulation result showed that the current $i_{2}$ is about 1.1 Arms which is the minimum propagation current of the wire. Then, it can be assumed that the temperature distribution profile of the normal zone is almost uniform along the superconducting wire. The joule heat loss per unit length at the normal zone balances with the heat flux at the surface of the normal zone of the wire (thermally equilibrium state). Therefore, "Recovery Time" is independent of the limiting current after a sufficient time from the fault occurrence.

\section{CONCLUSION}

The recovery process of transformer type SCFCL was investigated by experiments and computer simulation using threephase SCFCL. The results obtained are as follows. The "Recovery Time" decreases, as the "Fault Time" is longer. It is pointed out that the FCL current at the limiting mode affects the cooling process of the secondary wire during the current limiting operation. The simulation results of the "Recovery Time" agree well with the experimental ones. The temperature of the normal zone was estimated by use of the simulation. It decreases even in the current limiting mode and its decreasing rate depends on the limiting current. After a long limiting operation (over $5 \mathrm{~s}$ ), the temperature saturated about $22 \mathrm{~K}$ and its distribution profile for the longitudinal direction is almost uniform. The secondary coil current is the minimum propagation current of the wire and the "Recovery Time" is quite short and independent of the limiting current.

\section{REFERENCES}

[1] H. J. Boenig and D. A. Paice, "Fault Current Limiter using a superconducting coil," IEEE Trans. Magn., vol. 19, no. 3, pp. 1051-1053, May 1983.

[2] B. Gromoll et al., "Resistive fault current limiters with YBCO films-100 kVA functional model," IEEE Trans. Appl. Superconduct., vol. 9, no. 2, pp. 656-659, June 1999.

[3] E. Leung et al., "Design and development of a $15 \mathrm{kV}, 20 \mathrm{kA}$ HTS fault current limiter," IEEE Trans. Appl. Superconduct., vol. 10, no. 1, pp. 832-835, Mar. 2000.

[4] K. Fujikawa et al., "Experimental study on superconducting fault current limiter with adjustable trigger current level," in Proc. 15th Int. Conf., Sept. 1998, pp. 571-574.

[5] Y. Shirai et al., "Study on recovery time of a superconducting fault current limiter with adjustable trigger current level," IEEE Trans. Appl. Superconduct., vol. 11, no. 1, pp. 2086-2089, Mar. 2001.

[6] H. Hatta, T. Nitta, S. Muroya, Y. Shirai, and T. Kitagawa, "Experimental study on sudden-short-circuit characteristic of synchronous generator with SCFCL," IEEE Trans. Appl. Superconduct., vol. 11, no. 1, pp. 2343-2346, Mar. 2001. 
[7] K. Fujikawa, Y. Shirai, T. Kitagawa, M. Shiotsu, T. Nitta, and H. Hatta, "Study on degradation of trigger current level of superconducting fault current limiter of transformer type," IEEE Trans. Appl. Superconduct., vol. 11, no. 1, pp. 2410-2413, Mar. 2001.

[8] Thermal Conductivity: Metallic Elements And Alloys: IFI Plenum, 1970, pp. 561-567.

[9] Specific Heat: Metallic Elements And Alloys: IFI Plenum, 1970, pp. 341-345.
[10] Data Book On Cryogenic Engineering (in Japanese): Kansai Branch, The Cryogenic Society of Japan, 1997, vol. VI-7.

[11] Y. Shirai et al., "Recovery characteristics of fault current limiter with adjustable trigger current level," IEEE Trans. Appl. Superconduct., vol. 9, no. 2, pp. 1381-1384, June 1999. 